See discussions, stats, and author profiles for this publication at: https://www.researchgate.net/publication/328726200

\title{
Considerations for the acquisition and inversion of NMR T2 data in shales
}

Article in Journal of Petroleum Science and Engineering · November 2018

DOI: 10.1016/j.petrol.2018.10.109

CITATIONS

0

2 authors:

a

Nadia Testamanti

Curtin University

11 PUBLICATIONS 20 CITATIONS

SEE PROFILE
READS

89

Reza Rezaee

Curtin University

186 PUBLICATIONS 1,477 CITATIONS

SEE PROFILE

Some of the authors of this publication are also working on these related projects:

Reservoir Characterization for $\mathrm{CO} 2$ Injectivity and Flooding in Petroleum Reservoirs Offshore Malaysia View project

Petrophysical rock typing View project 


\section{Accepted Manuscript}

Considerations for the acquisition and inversion of NMR $\mathrm{T}_{2}$ data in shales

M. Nadia Testamanti, Reza Rezaee

PII: $\quad$ S0920-4105(18)30977-X

DOI: $\quad$ https://doi.org/10.1016/j.petrol.2018.10.109

Reference: PETROL 5472

To appear in: Journal of Petroleum Science and Engineering

Received Date: 29 May 2018

Revised Date: 26 October 2018

Accepted Date: 30 October 2018

Please cite this article as: Testamanti, M.N., Rezaee, R., Considerations for the acquisition and inversion of NMR T 2 data in shales, Journal of Petroleum Science and Engineering (2018), doi: https:// doi.org/10.1016/j.petrol.2018.10.109.

This is a PDF file of an unedited manuscript that has been accepted for publication. As a service to our customers we are providing this early version of the manuscript. The manuscript will undergo copyediting, typesetting, and review of the resulting proof before it is published in its final form. Please note that during the production process errors may be discovered which could affect the content, and all legal disclaimers that apply to the journal pertain. 


\title{
Considerations for the Acquisition and Inversion of NMR $\mathrm{T}_{2}$ Data in Shales
}

\author{
M. Nadia Testamanti ${ }^{\mathrm{a}, *}$, Reza Rezaee ${ }^{\mathrm{a}}$ \\ ${ }^{a}$ Curtin University \\ Perth, Western Australia
}

\begin{abstract}
Low-field Nuclear Magnetic Resonance (NMR) is a non-invasive method widely used in the petroleum industry for the evaluation of reservoirs. Pore structure and fluid properties can be evaluated from transverse relaxation $\left(\mathrm{T}_{2}\right)$ distributions, obtained by inverting the raw NMR signal measured at subsurface conditions or in the laboratory. This paper aims to cast some light into the best practices for the $\mathrm{T}_{2}$ data acquisition and inversion in shales, with a focus on the suitability of different inversion methods. For this purpose, the sensitivity to various signal acquisition parameters was evaluated from $\mathrm{T}_{2}$ experiments using a real shale core plug. Then, four of the most common inversion methods were tested on synthetic $\mathrm{T}_{2}$ decays, simulating components often associated with shales, and their performance was evaluated. These inversion algorithms were finally applied to real $\mathrm{T}_{2}$ data from laboratory NMR measurements in brine-saturated shale samples. Methods using a unique regularization parameter were found to produce solutions with a good balance between the level of misfit and bias, but could not resolve adjacent fast $\mathrm{T}_{2}$ components. In contrast, methods applying variable regularization - based on the noise level of the data - returned $\mathrm{T}_{2}$ distributions with better accuracy at short times, in exchange of larger bias in the overall solution. When it comes to reproducing individual $\mathrm{T}_{2}$ components characteristic of shales, the Butler-Reeds-Dawson (BRD) algorithm was found to have the best performance. In addition, our findings suggest that threshold $T_{2}$ cut-offs may be derived analytically, upon visual inspection of the $T_{2}$ distributions obtained by two different NMR inversion methods.
\end{abstract}

Keywords: Formation Evaluation, Nuclear Magnetic Resonance, Transverse Relaxation, Porous Media, Inversion, Shale

\footnotetext{
${ }^{*}$ Corresponding author

Email address: m.testamanti@postgrad.curtin.edu.au (M. Nadia Testamanti)
} 


\section{Introduction}

Nuclear Magnetic Resonance (NMR) is an established technique that has applications across a wide range of scientific disciplines, including medicine, material science and chemistry. One of the key features of this method is that measurements are non-invasive, so NMR is routinely used in the study of fluid-saturated porous media. This has proven particularly useful to the petroleum industry, where NMR tools are routinely used for the evaluation of reservoirs.

The analysis of NMR data obtained from wireline logging and laboratory measurements purpose, both experimental results from NMR measurements on shales and simulated $T_{2}$ curves will be analyzed. The $T_{2}$ data presented in Testamanti \& Rezaee (2017) will then be reprocessed to illustrate how the concepts revised in the current work could be applied for the analytical determination of threshold $T_{2}$ cut-offs, which will be compared with the

on cores can yield valuable information about the pore system and the different fluids present (Dunn et al., 2002, Kenyon, 1997; Freedman, 2002; Prammer et al., 1996, Coates et al. 1998). One-dimensional experiments are the most common application of the NMR technique for the study of porous media, and involve $T_{1}$ and $T_{2}$ measurements using lowfield NMR spectrometers. Relaxometry experiments inspect the characteristic relaxation of the rock and fluids within its pores, which can be used to assess porosity and pore size distributions. However, a robust quantitative analysis of NMR results is essential before reliable petrophysical parameters can be derived. The narrow pores and fast relaxation rates characteristic of NMR measurements in shales generates additional challenges in NMR data acquisition and interpretation. that can yield useful petrophysical information. Previous studies have suggested different approaches for the application of the NMR technique in shales (Washburn et al., 2015), but a universal standard is still lacking. This paper will examine the influence of various methodologies on the results obtained from laboratory NMR measurements in shales, aiming to cast some light on the best practices for $T_{2}$ data acquisition and inversion. For this findings from our experimental study. 


\section{NMR Theory}

Nuclear magnetic resonance can yield valuable information about the petrophysical characteristics of a reservoir and the different fluids present. Relaxometry experiments, in particular, are used in reservoir rocks to measure longitudinal $\left(T_{1}\right.$ and transverse relaxation $\left(T_{2}\right)$. The measurement of $T_{2}$ using the Carr-Purcell-Meiboom-Gill (CPMG) sequence is preferred over $T_{1}$ in porous media, mainly due to the duration of experiments and the difficulties associated with $T_{1}$ data acquisition through well-logging (Kleinberg et al., 1993).

The $T_{2}$ relaxometry technique involves an initial polarization of the nuclei in the direction of the imposed magnetic field $\left(B_{0}\right)$, followed by the application of a series of radiofrequency $(\mathrm{RF}$ pulses. The decaying signals emitted by the precessing nuclei, are then measured. Since the magnetization is induced on hydrogen protons, NMR logging tools are considered to yield lithology-independent results, thus avoiding the pitfalls of traditional wireline logging methods (Kenyon, 1997, Anovitz \& Cole, 2015). In saturated porous media, the $T_{2}$ relaxation rates are a function of individual intrinsic (or bulk), surface and diffusion relaxation processes, governed by the equation:

$$
\frac{1}{T_{2}}=\frac{1}{T_{2 \mathrm{~b}}}+\rho \frac{S}{V}+\frac{D\left(\gamma G T_{E}\right)^{2}}{12}
$$

where $T_{2 \mathrm{~b}}$ is the bulk fluid transverse relaxation, $\rho$ is the surface relaxivity, $S / V$ is the surface-to-volume ratio of the pore, and the last term represents the diffusion induced transverse relaxation, controlled by the molecular self-diffusion coefficient $(D)$, the magnetic field gradient $G$ ) and the gyromagnetic ratio of the precessing nuclei (Bloembergen et al. 1948).

\subsection{Data Acquisition}

The CPMG Pulse Sequence

The CPMG sequence involves an initial $90^{\circ} \mathrm{RF}$ pulse that tips the magnetization into the transverse plane. The loss of coherence suffered by the nuclei will induce a free induction decay (FID). After a time equivalent to half an echo-spacing $\left(T_{E}\right)$, a $180^{\circ}$ pulse will refocus the spins and the spin-echo will be recorded by the NMR tool. This will then be followed by a series of $180^{\circ}$ pulses, applied at an interval $T_{E}$, which will produce a spin-echo train, as 


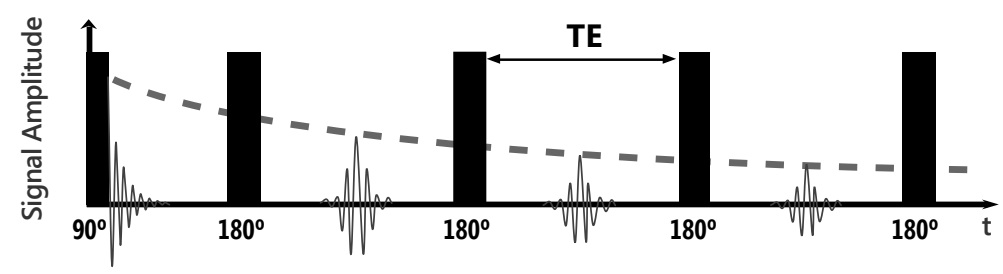

Figure 1: Schematic of the CPMG pulse sequence, used to generate an echo train during $\mathrm{T}_{2}$ measurements (dashed line). $\mathrm{T}_{E}$ is the echo-spacing (i.e. time between consecutive $180^{\circ}$ pulses).

illustrated in Fig. 1. It is worth noting that the dephasing caused by molecular interactions and diffusion cannot be reversed or avoided, so the CPMG pulse sequence can only be used to offset the loss of coherence resulting from $B_{0}$ inhomogeneities (Coates et al., 1999). Once a complete refocusing of all nuclei is no longer possible and the loss of coherence becomes irreversible, the spin-echo train will decay. $T_{2}$ may thus be defined as the time it takes for the nuclear spins to lose coherence with one another, and may either be shorter or equal to $T_{1}$. The $T_{2}$ decays are the primary target of NMR logging tools, from which $T_{2}$ distributions are derived by signal inversion.

\subsection{Signal Processing}

In NMR measurements, the decaying signals decomposed in the time domain describe a Laplace transform, which must be inverted to obtain the distribution of characteristic $T_{2}$ rates. Different approaches can be used in solving the NMR inversion problem, so the use of a suitable method is crucial for obtaining $T_{2}$ distributions that can yield meaningful petrophysical information.

In the absence of experimental errors or noise, an ideal inversion problem can be represented by a linear relationship $Y=A X$, where $Y$ is the measured data for a given system, $A$ is the operator describing the physics of the problem, and $X$ includes the model parameters to estimate. Experimental noise $(\xi)$ cannot be suppressed from real NMR measurements and, consequently, measured decaying NMR signals $m(t)$ have a structure similar to a Fredholm integral equation of the first kind (Polyanin \& Manzhirov, 2008), as seen below:

$$
m(t)=\int k\left(t, T_{2}\right) s\left(T_{2}\right) d T_{2}+\xi
$$


where $k\left(t, T_{2}\right)$ is the evaluation matrix (or kernel), $s\left(T_{2}\right)$ is the unknown spectrum. The determination of $s\left(T_{2}\right)$ is the key objective of the $T_{2}$ inversion process.

The integral in Eq. (2) acts as a low-pass filter that returns the smooth decaying signal $m(t)$, by attenuating the high-frequency components in $s\left(T_{2}\right)$. These high frequency components, along with noise, will be amplified if $s\left(T_{2}\right)$ is directly estimated by the inversion of the recorded data $m(t)$. Small perturbations in the measured data could lead to great variations in the spectra obtained, and such solutions are therefore said to be unstable. Furthermore, multiple distributions could produce similarly shaped $T_{2}$ decays (and vice versa) in the $1 \mathrm{D}-\mathrm{NMR}$ inversion problem. Since the uniqueness and stability of solutions cannot be guaranteed, the NMR inversion problem is considered as ill-posed. In such cases, where a straightforward inversion to solve the problem stated in Eq. (2) is not possible, estimating the $T_{2}$ spectra requires a different approach that includes the use of regularization techniques (Polyanin \& Manzhirov, 2008). The inversion of $T_{2}$ signal decay must be transformed into a numerical optimization problem that can admit a unique solution, ensured by the introduction of constraints. The $T_{2}$ distribution will then be determined as the solution that best fits the measured data (under certain constraints).

\section{Discretisation}

The raw data recorded during NMR measurements is a complex signal, sampled at intervals $T_{E}$; however, only the real part of the NMR signal is generally used for further analysis in spectroscopy (Rutledge, 1996). The continuous integral in Eq. (2) can be transformed into a linear algebraic system by discretization, taking the form:

$$
M=K S+E
$$

where the vector $M \in \mathbb{R}^{m}$ contains the data acquired at times $t_{i}, K \in \mathbb{R}^{m \times n}$ is the kernel, $S \in \mathbb{R}^{n}$ is the distribution evaluated for the relaxation times $T_{2 j}$, and $E \in \mathbb{R}^{m}$ is the error (or noise). In saturated rocks, the relaxation components are assumed to decay exponentially, so Eq. (3) can be rewritten as:

$$
m_{i}=\sum_{j=1}^{N} s_{j} e^{-\left(t_{i} / T_{2 j}\right)} \quad i=1,2, \ldots, M
$$


where the relaxation times $T_{2 j}$ are typically logarithmically spaced (Borgia et al., 1990,

Whittall, 1996).

\section{Singular Value Decomposition}

The stability and computing cost of the NMR inversion problem can be improved by projecting the data onto a subspace, defined from a truncated singular value decomposition (SVD) of the kernel matrix (Mitchell et al., 2012; Hürlimann \& Venkataramanan, 2002, Song et al., 2002; Venkataramanan et al., 2002). The matrix $K$ can then be factorized by means of SVD:

$$
K=U \Sigma V^{T}
$$

where $U \in \mathbb{R}^{m \times m}$ and $V \in \mathbb{R}^{n \times n}$ are orthogonal singular matrices, and $\Sigma \in \mathbb{R}^{m \times n}$ is a diagonal matrix of the singular values in descending order (Hansen, 1987).

The next step involves the compression of data by truncating the singular values in $\Sigma$. The inclusion of a large number of values enhances the effect of noise in the spectrum, but limiting their number when the signal-to-noise ratio (SNR) is low may lead to solutions with artificially broad peaks (Song et al., 2005). Thus, only the first components of $\Sigma$ that hold significant information should be retained, while those that contain mostly noise are to be discarded. The number of significant singular values $\tilde{n}_{r}$ depends on the condition number, calculated as the ratio of the largest to smallest singular values in the rank reduced $\Sigma_{r}$. The truncation level is commonly determined based on the signal-to-noise ratio of the data (Mitchell et al., 2012, Song et al., 2005), and should satisfy the necessary conditions to ensure well-conditioned matrices (Hansen, 1987). The truncated matrices will be $U_{r}(m \times$ $r), \Sigma_{r}$ (diagonal, $\left.r \times r\right), V_{r}(n \times r)$. The compressed data vector will then be calculated as $M_{r}=U_{r}^{T} M$, and the reduced kernel is $K_{r}=\Sigma_{r} V_{r}^{T}$.

The truncated SVD approach can be used for regularization, but requires previous knowledge about the solution to identify the optimal truncation value and cannot guarantee positive solution elements, and should thus be used in combination with other methods (Venkataramanan et al. 2002). 

problem described by Eq. (6) is then extended to:

$$
\underset{S_{r} \geqslant 0}{\arg \min }\left\|M_{r}-K_{r} S_{r}\right\|_{2}^{2}+\lambda\left\|L S_{r}-S_{r 0}\right\|_{2}^{2}
$$

where $L$ is the regularization operator (matrix of additional constraints), and $\lambda$ is a variable known as the regularization parameter. The $L$ operator can regularize the solution via 
an $L_{0}, L_{1}$ or $L_{2}$ norm penalty function, often referred to as norm (or energy), slope or curvature smoothing (Dunn et al., 1994). The tuning parameter $(\lambda)$ in Eq. (7) regulates the smoothness of the solution relative to the misfit term. Larger values of $\lambda$ will dampen the smallest significant singular values (associated with noise) and thus lead to smoother solutions. The incorrect selection of the regularization parameter $\lambda$ can result in either under or over-smoothed spectra, so several methods have been developed to find the optimal $\lambda_{\text {opt }}$ that provides the best trade-off between misfit and smoothness of solutions to return realistic $T_{2}$ distributions.

The Tikhonov regularization described by Eq. (7) may be implemented using a direct (forward) or an indirect approach. The direct methodology involves an initial analysis to determine the optimal regularization parameter which is later used to calculate the best possible solution, often using the NNLS-LH method (Lawson \& Hanson, 1974). In contrast, the indirect approach finds the solution to the NMR problem with the help of auxiliary functions, such as the method proposed by Butler et al. (1981). Four methods for determining $\lambda_{\text {opt }}$ are presented below: the $L$-curve, $G C V, B R D$ and UPEN.

\section{Choice of Regularization Parameter}

The L-curve method, proposed initially by Lawson \& Hanson (1974) and further developed by Hansen (1992) and Hansen \& O'Leary (1993), was one of the first algorithms introduced to solve the NMR inversion problem. The plot of the solution norm versus its corresponding residual norm, as a function of a given range of $\lambda$ values results in an L-shaped curve as illustrated in Fig. 2, giving its name to this method. The point at which the slope of the curve abruptly changes is then selected as the optimal smoothing coefficient $\lambda_{\text {opt }}$. The selection of the best regularization parameter may thus be somewhat arbitrary with the L-curve method, depending on the data analyzed.

The generalized cross-validation (GCV) method, proposed by Golub et al. (1979), finds the optimal regularization parameter $\lambda_{\text {opt }}$ that minimizes the function:

$$
G C V\left(\lambda_{\text {opt }}\right)=\frac{\left\|M_{r}-K_{r} S_{r, \lambda}\right\|_{2}^{2}}{\left[\operatorname{trace}\left(\mathbb{I}-S_{r, \lambda}\right)\right]^{2}}
$$

where $\mathbb{I}$ is the identity matrix, and $S_{r, \lambda}$ is the solution calculated for a certain smoothing 
coefficient $入$

In the Butler-Reed-Dawson (BRD) method, an auxiliary convex function is minimized to find the $\lambda_{\text {opt }}$ (instead of performing direct iterations over $c_{r}$ ) based on the discrepancy principle (Butler et al., 1981; Morozov, 1984). The regularization parameter may be empirically determined as a function of a specified level of misfit in the data, such as RMSE. It is also common for solutions to be obtained based on the SNR of the data, generally defined as $\mathrm{SNR}=M(0) / \sigma$, where $\sigma$ is the noise standard deviation (Song et al. 2005). Using the Newton method, the estimated $\lambda$ values are updated stepwise as a function of $190 \sqrt{n_{r}} /\left\|c_{r}\right\|$ (Mitchell et al., 2012), and the misfit error is calculated as:

$$
\chi\left(c_{r}, \lambda\right)=\frac{\lambda\left\|c_{r}\right\|}{\sigma}
$$

The S-curve is commonly used to find the optimal regularization parameter, which evaluates the sigmoidal shape displayed in the log-log graph of the misfit error, as a function of $\lambda$ (Fordham et al. 1995). The optimal solution is found at the "heel" of the S-curve, where $\lambda$ satisfies:

$$
\frac{d\left(\log \chi\left(c_{r}, \lambda\right)\right)}{d(\log \lambda)}=\text { TOL }
$$
smoothing. 


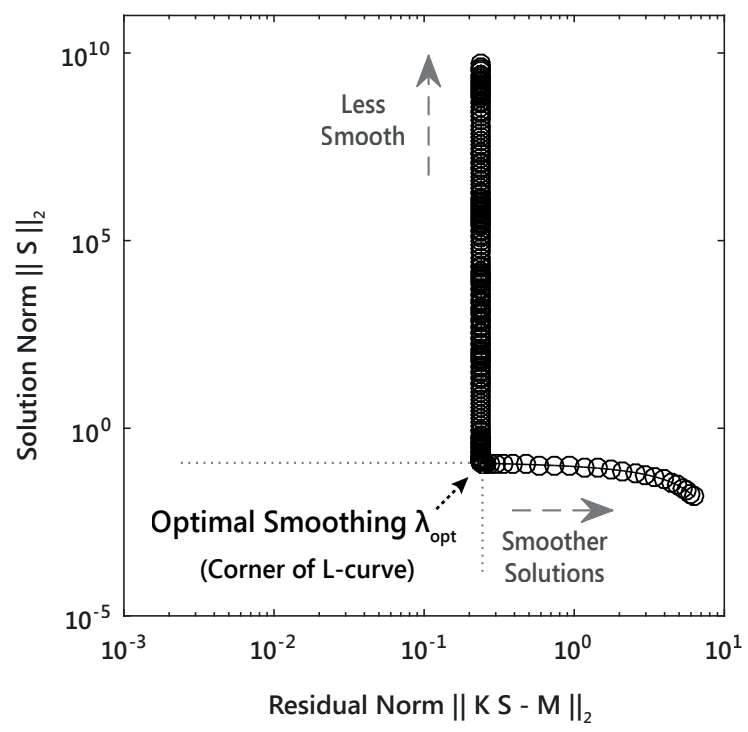

Figure 2: Graphical analysis of the solution norm $\|s\|_{2}$, calculated for discrete regularization parameter values $(\lambda)$ and plotted against the corresponding residual norm $\|K S-M\|_{2}$, known as the L-curve method. The horizontal part of the curve indicates the residual norms that are most sensitive to errors arising from the incorrect selection of $\lambda$. The vertical part illustrates the solution norms that are most sensitive to errors associated with amplification of random noise. The optimal $\lambda_{\text {opt }}$ is found at the corner of the discrete Lcurve, either visually or based on a numerical method such as the adaptive pruning algorithm (Hansen 2015 .

\section{Methodology}

Experimental results from NMR measurements conducted in shales and simulated $T_{2}$ film to prevent moisture evaporation or absorption. The sample belongs to the Carynginia Formation, a dry gas-prone shale section found in most of the northern Perth Basin (Mory \& Iasky, 1996). 
The inversion methods included in the previous section of this paper are tested on synthetic $T_{2}$ data, as shown in Subsection 4.2 . The $T_{2}$ decays were generated to replicate the equivalent relaxation response that would be obtained from measurements conducted in real shale samples. The Lexus module in the Prospa software (Magritek) was used for the BRD inversions, whereas the NNLS-LH/L-curve, NNLS-LH/GCV and UPEN algorithms were implemented in Matlab (Mathworks), using purposely written code. In the latter case, final computations were done using the NNLS function included in the MERA Toolbox for Matlab (Does, 2014).

In Subsection 4.4, the four inversion methods are then tested on real NMR data. The raw $T_{2}$ decays were originally obtained from brine-saturated core plugs, in the experimental study presented in Testamanti \& Rezaee (2017). The $T_{2}$ decays were acquired using 10,000 echoes and a 100 ps echo time, with the magnet temperature set to $30{ }^{\circ} \mathrm{C}$. The number of scans was automatically adjusted to collect data with a minimum SNR of 200 and the background signal - corresponding to the probe and plastic wrap - was subtracted from the total measured $T_{2}$ decays. The raw data was phased using the Prospa software 230 (Magritek) to maximize the contribution from the real component in the signal, later used for inversion. More information on the geological background of these six samples and preparation procedures can be found in the original paper (Testamanti \& Rezaee, 2017).

\section{Results and Discussion}

\section{1. $T_{2}$ Data Acquisition}

235 As reviewed in the previous section, the CPMG sequence involves an initial polarization of the nuclear spins, followed by the application of a series of magnetic pulses $N_{E}$, spaced by a $T_{E}$ time. Consecutive CPMG pulse trains are separated by a wait time or interexperimental delay $\left(T_{W}\right)$, during which the polarization of the system builds up. The parameters $N_{E}, T_{E}, T_{W}$, as well as the pulse specifications, are all necessary inputs of $T_{2}$ CPMG experiments and must be suitably chosen by the user. The output will be a vector with the raw $T_{2}$ data, recorded at fixed intervals $T_{E}$. The impact of various acquisition parameters on results is discussed below. 


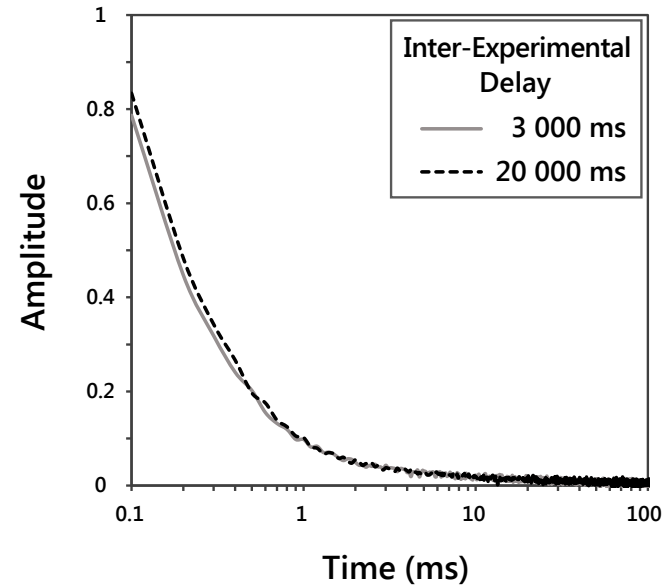

(a)

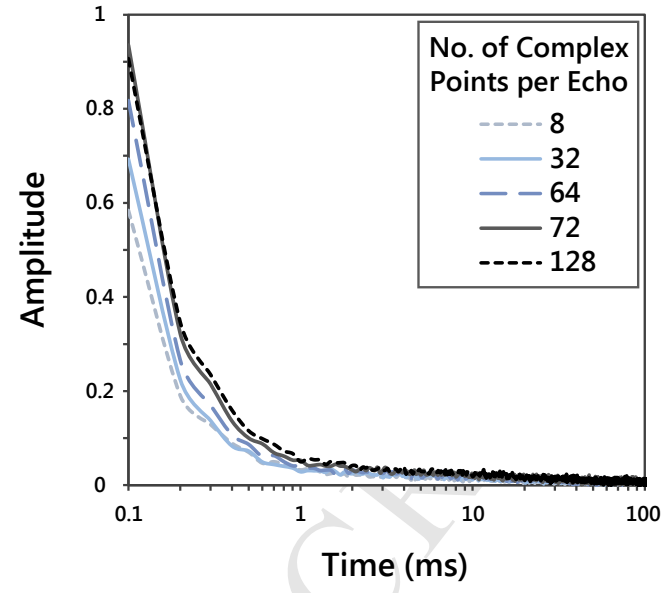

(b)

Figure 3: $\mathrm{T}_{2}$ decay curves showing (a) the effect of the inter-experimental delay parameter on $\mathrm{M}(0) ;(\mathrm{b})$ the influence of the number of complex points sampled per echo on signal quality.

\section{Inter-Experimental Delay}

The magnitude of $M_{0}$ is estimated from the signal amplitude measured after the first $\mathrm{RF}$ pulse in the sequence. Thus, $T_{W}$ should be set to at least three times the expected $T_{1}$ value, to allow a $95 \%$ polarization of the spins and avoid underestimating $M_{0}$. Shales have very fast relaxation rates, so generally short $T_{W}$ times can be safely used for $T_{2}$ measurements. This is illustrated in Fig. ??, where the impact of the inter-experimental delay parameter on the measured $M_{0}$ of a shale core sample is examined. The $T_{2}$ decays were acquired with the P54 probe for 3,000 echoes, with a $T_{E}=100 \mu$ s and the same target $\mathrm{SNR}=220$ but using short and long $T_{W}$ values, set to 3,000 and 20,000 ms respectively. Similarly shaped $T_{2}$ decay curves emerge from the graph, while the difference between the calculated $M_{0}$ was about $7 \%$. The use of longer inter-experimental delays was thus found to only marginally improve the estimation of $M_{0}$ values. The impact of the $T_{W}$ parameter on the overall duration of measurements, however, becomes more significant in shales and should be evaluated against time constrains if applicable.

\section{Signal-to-Noise Ratio}

Despite being relatively weak, NMR signal can be detected in rocks owing to the abundance of hydrogen protons in the reservoir fluids. Improving the quality of NMR data 
measurements can be seen as equivalent to reducing the experimental noise, which improves the effective SNR of the data. After signal is detected, the in-phase and quadrature signals for each spin-echo are sampled, according to the number of complex points and dwell time set by the user, and digitized in the spectrometer before returning to the computer (Kleinberg, 1999). To enhance the quality of the results, a signal averaging - or stacking - process is performed on the raw data, followed by a phase correction. The final outputs are two data vectors, one containing the real components of the spin-echo train from which $T_{2}$ distributions will be obtained, and another containing the imaginary portion of the complex signal that represents the noise with zero mean (Coates et al., 1999).

The acquisition of a large number of echoes $N_{E}$ allows the decay of real components in the signal to noise level, ensuring the detection of all the $T_{2}$ relaxation rates present in the core sample. In practice, the experimental settings should satisfy the condition $N_{E}$ $\geq T_{2, \max } / 3 T_{E}$ to detect the full range of $T_{2}$ components (Coates et al. 1999$)$. As observed in Fig. ??, shales have fast relaxation rates so most of the signal should be captured within the first few hundred milliseconds.

275 The SNR of the acquired data may be enhanced by performing a signal averaging or stacking. This technique relies on the random feature of noise, as opposed to the reproducibility of the NMR signal, and therefore cannot filter out coherent noise. By averaging the signal from a number of successive scans $N$, the amplitude will grow at a rate of $N$ whereas the growth rate of noise will be $\sqrt{N}$. The SNR improvement will then be proportional to $\sqrt{N}$ averaged, where the number of scans should be a multiple of 4 for a full phase cycling. Another method often used to enhance the SNR consists in increasing the number of complex points sampled per echo. The product of this sampling number (ideally a power of 2$)$ and the dwell time will determine the total acquisition time $\left(T_{\text {acq }}\right)$ for each spin-echo; the spectral width is given by the reciprocal of the dwell time. The minimum $T_{E}$ available for the instrument will be limited by the acquisition time, and should be greater than $T_{\text {acq }}$ plus an added delay to avoid artifacts in the signal due to the acoustic ringing of the probe. Nonetheless, the law of diminishing returns applies to both of the SNR enhancement techniques previously described and should be carefully considered when experimental parameters are selected. The acquisition of a large number of points per echo may overheat the $\mathrm{RF}$ amplifier and thus damage the instrument, while an increase in the 
number of scans can extend the duration of experiments considerably. Fig. $3 \mathrm{~b}$ shows the $T_{2}$ curves obtained from CPMG sequences on a real shale core sample, where the raw signal was sampled using a variable number of complex points. Measurements were conducted using Magritek's P29 probe, with 2,000 echoes and a $T_{E}=100$ ps. Increasing the number of complex points sampled per echo from 8 to 32 leads to a $15 \%$ rise in the initial signal amplitude, similar to increasing the sampled points from 32 to 64, and then from 64 to 72. No substantial variations are observed between the curves acquired with 72 and 128 points per echo. The proportional rise in the noise standard deviation, however, prompts to question whether the increase in the first few data points could be in fact an artificial enhancement, caused by acoustic ringing effects (Gerothanassis, 1987). The use of a long acquisition delay is often recommended to prevent the collection of spin-echoes distorted by the probe free ringing, but that could also lead to the loss of valuable information in tight porous media. Since most of the signal decay generally occurs in the first few data points, the use of post-acquisition filters and alternative data fitting techniques that can compensate for offset and acoustic ringing effects may be more suitable for processing NMR data in shales (Freedman, 2002; Venkataramanan et al., 2013; Salimifard et al., 2017).

Alternatively, the use of relatively larger core samples, where hydrogen nuclei are more abundant, can yield an improvement of the signal quality. Since this is not always possible, the use of instruments with smaller RF coils could be another option for achieving higher SNR levels during measurements.

\section{Echo-Spacing $\left(T_{E}\right)$}

The use of shorter $T_{E}$ enables the earlier detection of NMR signal, desirable in the case of rocks with fast relaxing components. The number of complex points sampled per spin-echo, the pulse length and the acquisition bandwidth - along with the instrument's characteristics - will limit the minimum echo time that can be used for measurements. SNR will generally increase with decreasing $T_{E}$, as more data points will be generated (and recorded) at shorter intervals. The reduction in $T_{E}$ also has the unintended consequence of accentuating acoustic ringing effects. The first echoes are generally more affected by the acoustic ringing of the probe which would consequently become part of the recorded signal, causing a very high initial amplitude followed by a coherent transient (Coates et al. 
1999). This effect can be observed in Fig. 4a, where the background signal acquired with a $T_{E}$ of 40 us shows a higher initial amplitude, compared to the value obtained at a 100 us echo-spacing.

The ringing noise can be filtered out to some extent by manipulating the digital filter bandwidth, at the expense of loss in sensitivity owing to the limitations on the minimum $T_{E}$ possible (Salimifard et al., 2017), or attenuated by the use of smaller probes. The effect of random noise can be mitigated by simply subtracting the NMR signal corresponding to the instrument, from measurements on core samples. While background noise generally has little impact on results from high porosity rocks, it can contribute substantially to the NMR signal measured in shales (Saidian, 2015, Salimifard et al., 2017). It is therefore good practice to obtain the background signal emitted by sample containers or plastic wraps and by the instrument itself, which should be then subtracted from the $T_{2}$ decays - prior to data inversion. The effect of subtracting the background signal from $T_{2}$ measured on a real shale core sample is illustrated in Fig. 4b, where experiments were conducted at $40 \mu \mathrm{s}$ and 100 us echo-spacings using a P29 probe. A greater portion of the fast $T_{2}$ components could be detected using a shorter $T_{E}$, although this difference becomes less significant beyond $0.5 \mathrm{~ms}$. These fast $T_{2}$ relaxation rates are typically associated with organic and inorganic micropores, so short echo-spacings should be used whenever possible to investigate the full range of pore size ranges found in the shale matrix.

340 A final point to consider is the issue of thermodynamic stability, crucial for $T_{2}$ measurements based on the CPMG pulse sequence. In core samples where narrow pores are abundant, the duration of experiments can increase considerably and span several hours. Failure to achieve isothermal conditions could lead to the underestimation of $M_{0}$, so core samples should always be allowed to stabilize to the magnet temperature prior to measurements. Alternatively, samples could be pre-heated before loading them into the spectrometer, provided that they are tightly sealed to avoid unintended fluid loss due to vaporisation. 


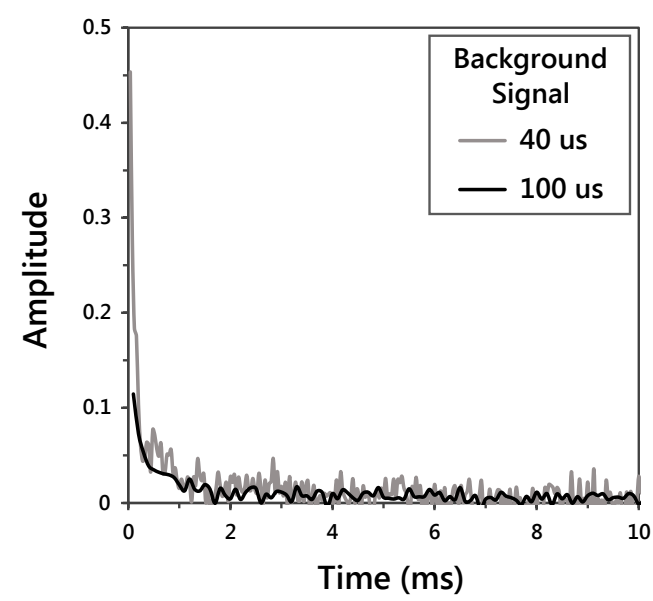

(a)

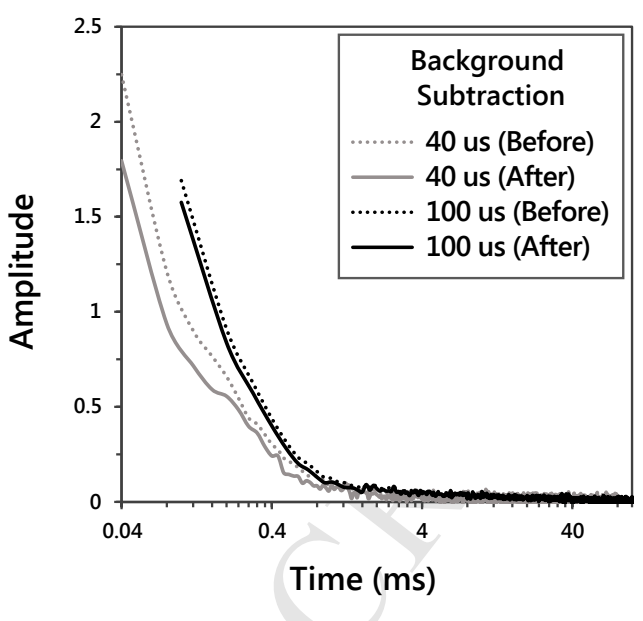

(b)

Figure 4: $\mathrm{T}_{2}$ decay curves showing (a) the background signal measured; (b) the effect of removing the background noise from the main signal, acquired at different echo-spacing. Measurements conducted using Magritek's P29 probe.

\section{2. $T_{2}$ Data Inversion}

The ultimate goal of the $T_{2}$ inversion process is finding the spectrum that best represents the real relaxation mechanisms in the saturated porous media. Most NMR inversion methods can correctly find solutions for systems where the dominant $T_{2}$ components are sufficiently separated, so the choice of the inversion algorithm is not a significant source of concern. This, however, is not usually the case in complex porous systems with abundant small pore sizes such as shales. The shale matrix typically has a substantial clay and organic content, with protons susceptible to magnetization that yield fast $T_{2}$ relaxation rates, often generating most of the total NMR signal acquired. Inversion algorithms must be therefore carefully selected, as the reconstructed signal should include sharper peaks at short $T_{2}$ times, which are associated with the relaxation of fluids in the smaller pores, clay bound water and solid organic matter present in shales. Caution must be nonetheless applied, to avoid artifacts in the spectrum at longer $T_{2}$ times that may not be representative of real nuclei relaxation within the larger pores.

The inversion algorithms described in the previous section were tested on a set of simulated $T_{2}$ decays emulating the relaxation rates commonly associated with sandstones and shales, designated as case $\mathrm{A}$ and $\mathrm{B}$ in this study. The $T_{2}$ curves were obtained from syn- 
thetic distributions with the following distinctive features: (A) three peaks, centred on 2, 14 and $44 \mathrm{~ms}$, displaying maximum normalized amplitudes of $20 \%, 50 \%$, and $30 \%$ respectively; (B) four peaks, centred on 0.1, 2, 14 and $44 \mathrm{~ms}$, displaying maximum normalized amplitudes of $20 \%, 40 \%, 30 \%$, and $10 \%$ respectively. The $T_{2}$ components used as input for the simulations are indicated with black lines in Fig. 5 . The $T_{2}$ decays were generated for 3,000 and 10,000 echoes, based on a 100 us echo time. Gaussian noise was also added to the raw $T_{2}$ decay curves, to obtain SNR levels comparable to those of the experimental NMR data presented in the previous subsection.

\section{Data Compression}

Before inversion, the size of acquired data is reduced for computational efficiency purposes and to filter out noisy values. The initial compression of NMR data can be computed with the TSVD method, which requires the selection of an optimal truncation value (Venkataramanan et al., 2002). Choosing this threshold incorrectly can lead to substantial attenuation of the shortest $T_{2}$ (Prammer, 1994; Song et al., 2005), and consequent loss of valuable information in systems where fast components are expected. If a large number of singular values are kept before inversion, the identification of individual adjacent peaks in the $T_{2}$ spectrum could be compromised and lead to distributions featuring one broad peak at short $T_{2}$ times. In shales, the earliest raw data points will likely contain the most valuable information and must be preserved, whereas the noisy part of the $T_{2}$ curve should be pruned before inversion. The truncation criteria also becomes more critical as the size of acquired data increases, relative to the time vector on which the inversion is evaluated. The data compression methodology can therefore have a more significant impact on results obtained in shales, compared to other types of reservoirs. In this study, the optimal number of significant singular values to preserve for the inversion were examined based on the L-curve method described in Hansen et al. (2007). The algorithm was implemented in Matlab (MathWorks), using the Regularization Toolbox (Hansen, 2015). For the simulated $T_{2}$ decays, a prune to between 25-50 significant values was generally found to produce stable solutions. 


\section{Inversion Method}

The incorrect selection of NMR inversion algorithms could potentially lead to gross interpretation errors, especially when the $T_{2}$ spectra are correlated to porosity and pore size distribution obtained from other core analysis methods. To assess the influence of inversion algorithms on shale results, the simulated $T_{2}$ curves were inverted for a logarithmically spaced vector with 200 points, within the range 0.01-600 ms for data simulated with 3,000 echoes, and between $0.01-2,000 \mathrm{~ms}$ for the cases with 10,000 echoes. Solutions were regularized with the energy of the $T_{2}$ spectrum, also know as norm-smoothing, except for inversions based on the UPEN method where curvature-smoothing was used instead. For the forward inversions using the NNLS-LH method, the optimal smoothing coefficients were chosen based on the L-curve (continuous blue line) and GCV criteria (light blue line), whereas distributions obtained by indirect approaches were computed with the BRD (dashed grey line) and UPEN algorithms (dotted grey line). The resulting $T_{2}$ distributions by the different methods are presented in Fig. 5, while the signal intensities are included in Table 1. The goodness of fit of the different inversion methods were evaluated by the sum of absolute error (SAE), root-mean squared error (RMSE), peak signal-to-noise ratio (PSNR) and structural similarity index (SSIM) (Wang et al., 2004; Miao et al., 2008); the residual statistics are summarized in Table 2.

For the case A, no significant differences are observed between the total signal simulated, recovered and calculated by the four NMR inversions, irrespective of the number of echoes (Table 1). In contrast, about $15 \%$ of the NMR signal in the simulated spectrum B is lost by the use of a $100 \mu$ s echo time (which allows only a partial detection of the peak centred on $0.1 \mathrm{~ms}$ ), while the acquisition of a larger number of echoes has a minor positive effect on the total amount of signal recovered. The comparison of case B results reveals that the BRD and UPEN algorithms can closely reproduce the original $T_{2}$ signal recovered (13.01 and 13.19 for 3,000 and 10,000 echoes, respectively), with an error $<0.5 \%$. The differences between the original and inverted signal intensities are greater for the NNLS-LH method, based on both the L-curve (1-2\%) and GCV criteria (2.5-4.5\%).

Fig. 5 shows that - in all cases - direct inversion approaches (NNLS-LH combined with L-curve and GCV methods) yields smoother $T_{2}$ spectra than the BRD and UPEN algorithms. In conventional reservoirs, smooth solutions featuring broad spectral peaks 
are generally considered to be physically representative of the saturated porous media, and therefore selected as the best fits. On the other hand, solutions including sharp peaks data disappear after the NNLS-LH/GCV inversion (cases A \& B), while the NNLS-LH/Lcurve algorithm returns seemingly better fits with more precise peak locations (case B). 

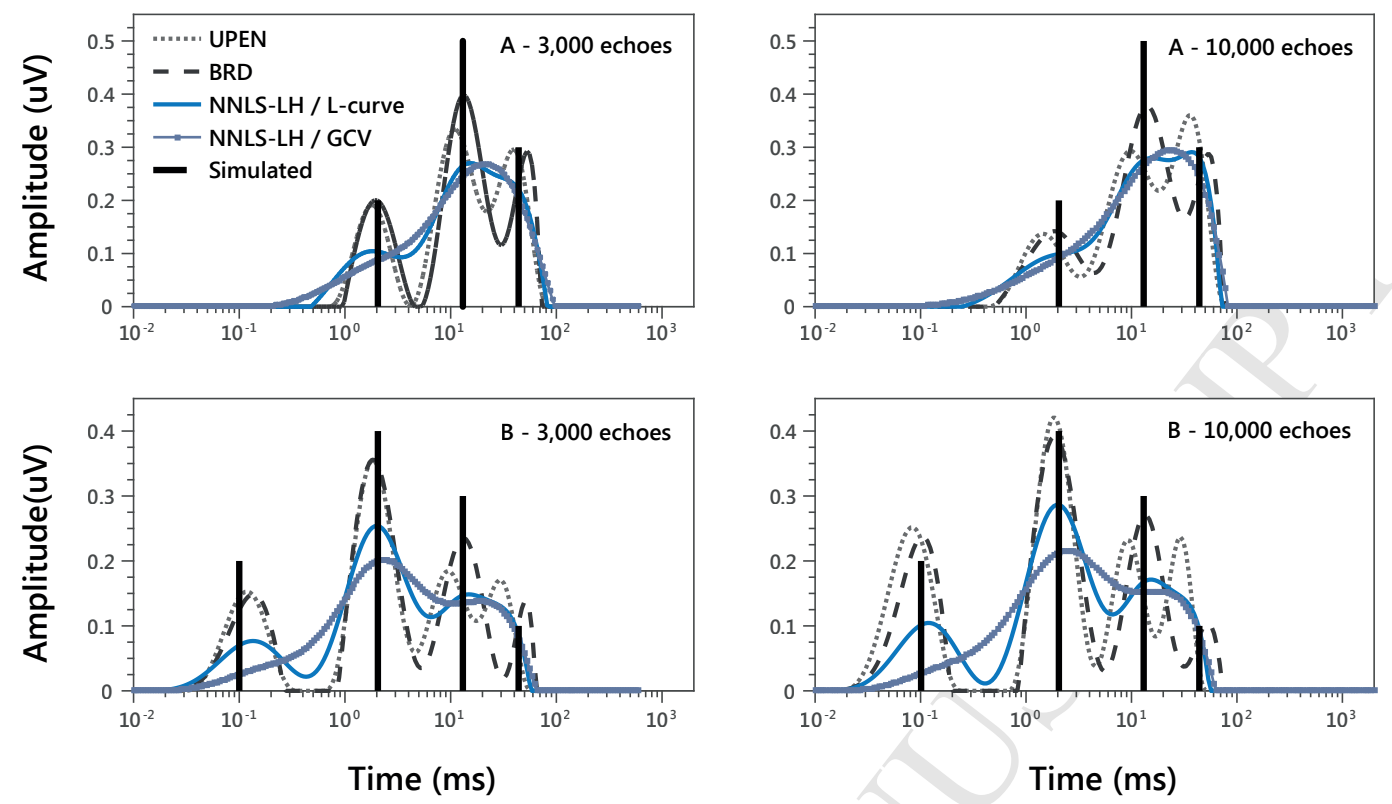

Figure 5: $\mathrm{T}_{2}$ spectra from simulated decay curves for 3,000 (left panel) and 10,000 echoes (right panel), inverted with the NNLS-LH/Lcurve, NNLS-LH/GCV, BRD and UPEN methods. The black vertical lines indicate the $\mathrm{T}_{2}$ components from which the decays were generated.

Interestingly, the NNLS-LH/L-curve produces larger residuals than the NNLS-LH/GCV inversion, and has poorer PSNR, RMSE and SSIM scores (Table 2). The global performance of the NNLS-LH/L-curve method seems to improve when the number of echoes acquired increases (case B), matching the inversion quality metrics of the NNLS-LH/GCV algorithm. On the other hand, indirect NMR inversion approaches return $T_{2}$ spectra displaying well-defined peaks. The BRD algorithm produces the best fits, in terms of both locating and estimating the amplitudes of the main peaks. The UPEN method is able to preserve the details of the original simulated data but fails to determine peak locations and amplitude correctly, while the weak associated PSNR, RMSE and SSIM scores render it the least reliable method out of the four herein analyzed. Overall, the best fits are obtained with the BRD method, as confirmed by the superior inversion quality metrics. Surprisingly, the acquisition of a larger number of echoes appears to negatively impact the $T_{2}$ inversions on the simulated dataset $\mathrm{A}$, while positive effects are observed on the dataset $\mathrm{B}$ results. 
Table 1: Comparison of NMR signal intensities for the simulated $\mathrm{T}_{2}$ data sets, obtained by the NNLS-LH with L-curve, NNLS-LH with GCV, BRD and UPEN inversion methods.

\begin{tabular}{llrrrrrr}
\hline Sample & $\begin{array}{l}\text { No. } \\
\text { Echoes }\end{array}$ & Original & Recovered & $\begin{array}{r}\text { NNLS-LH } \\
\text { (L-curve })\end{array}$ & $\begin{array}{r}\text { NNLS-LH } \\
\text { (GCV) }\end{array}$ & BRD & UPEN \\
\hline A & 3,000 & 13.69 & 13.44 & 13.46 & 13.45 & 13.45 & 13.42 \\
A & 10,000 & 13.69 & 13.47 & 13.48 & 13.42 & 13.51 & 13.45 \\
B & 3,000 & 15.32 & 13.01 & 12.85 & 12.67 & 13.01 & 12.95 \\
B & 10,000 & 15.32 & 13.19 & 12.96 & 12.64 & 13.18 & 13.14 \\
\hline
\end{tabular}

Table 2: Goodness of fit for the simulated NMR data sets, inverted with the NNLS-LH/L-curve, NNLSLH/GCV, BRD and UPEN methods. Quality of the inversion is assessed by the sum of absolute error (SAE), root-mean squared error (RMSE), peak signal-to-noise ratio (PSNR) and structural similarity index (SSIM). Errors are computed as the residuals between the initial data $(M)$ and the predicted decay curves from each method $\left(M^{p}=K S_{\text {reg }}\right)$. Low RMSE, and high PSNR and SSIM values are indicative of good quality inversions.

\begin{tabular}{|c|c|c|c|c|c|c|}
\hline Sample & $\begin{array}{l}\text { No. } \\
\text { Echoes }\end{array}$ & Original & $\begin{array}{l}\text { S-LH } \\
\text { urve) }\end{array}$ & $\begin{array}{r}\text { NNLS-LH } \\
(\text { GCV })\end{array}$ & BRD & UPEN \\
\hline & & \multicolumn{4}{|c|}{$\mathbf{S A E}\left(\times 10^{2}\right)$} & \\
\hline $\mathrm{A}$ & 3,000 & 8.22 & 1.57 & 1.50 & 1.46 & 1.71 \\
\hline $\mathrm{A}$ & 10,000 & 18.70 & 5.48 & 5.39 & 5.16 & 5.67 \\
\hline B & 3,000 & 4.15 & 1.67 & 1.63 & 1.39 & 1.82 \\
\hline \multirow[t]{2}{*}{ B } & 10,000 & 10.16 & 5.40 & 5.37 & 5.03 & 5.56 \\
\hline & & \multicolumn{4}{|c|}{ PSNR } & \\
\hline $\mathrm{A}$ & 3,000 & 241 & 234 & 238 & 241 & 227 \\
\hline $\mathrm{A}$ & 10,000 & 236 & 231 & 232 & 236 & 228 \\
\hline B & 3,000 & 245 & 229 & 231 & 244 & 222 \\
\hline \multirow[t]{2}{*}{ B } & 10,000 & 239 & 233 & 233 & 239 & 231 \\
\hline & & \multicolumn{5}{|c|}{ RMSE $\left(\times 10^{-2}\right)$} \\
\hline $\mathrm{A}$ & 3,000 & 41.12 & 6.76 & 6.46 & 6.24 & 7.33 \\
\hline $\mathrm{A}$ & 10,000 & 36.95 & 7.01 & 6.91 & 6.59 & 7.25 \\
\hline $\mathrm{B}$ & 3,000 & 21.83 & 7.13 & 7.02 & 6.02 & 7.72 \\
\hline \multirow[t]{2}{*}{ B } & 10,000 & 19.27 & 6.83 & 6.83 & 6.39 & 7.02 \\
\hline & & \multicolumn{5}{|c|}{ SSIM } \\
\hline $\mathrm{A}$ & 3,000 & 0.96 & 0.95 & 0.96 & 0.96 & 0.95 \\
\hline $\mathrm{A}$ & 10,000 & 0.89 & 0.88 & 0.88 & 0.89 & 0.88 \\
\hline $\mathrm{B}$ & 3,000 & 0.94 & 0.91 & 0.92 & 0.94 & 0.90 \\
\hline $\mathrm{B}$ & 10,000 & 0.88 & 0.87 & 0.87 & 0.88 & 0.86 \\
\hline
\end{tabular}




\subsection{Relaxation Mechanisms in Shales}

Hydrogen-rich particles confined to a bounded domain are often abundant in organic

470 would likely be an impossible task.

The degree of heterogeneity associated with shales, often displaying a wide separa- 

study (Testamanti \& Rezaee, 2017).

The $T_{2}$ decays were initially processed based on the NNLS-LH method, using the Lawson and Hanson module in the Prospa software (Magritek), by which the $T_{2}$ distributions the $T_{2}$ spectra (Watson \& Chang, 1997). Additionally, the intricate shale pore network may induce large internal magnetic field gradients and thus the Brownian motion of spins, leading to deviations from the fast diffusion regime (Hürlimann, 1998; Song, 2000, 2003 Washburn, 2014). These diffusion effects can be quantified based on laboratory 2D-NMR experiments, from which $T_{2}$ data corrections may be derived and the contribution from diffusion-induced signals estimated (Sun \& Dunn, 2004). Unfortunately, obtaining sound results from borehole measurements remains impractical in shale reservoirs with the technology currently available, owing to the high levels of noise and consequently low SNR, and $T_{2}$ experiments therefore remain as the most viable NMR data source to yield petrophysical information. One final point to consider is the impact of non-negligible internal gradients on the accuracy of NMR porosity determination, which may lead to its overestimation (McPhee et al., 2015). To minimize gradient-induced $T_{2}$ relaxation effects in shales, the use of the use of larger core samples, low field spectrometers and short echo-spacings in the CPMG sequence, is recommended.

\subsection{Comparison of NMR Inversion Methods for the Analytical Determination of Threshold} $T_{2}$ cut-offs

Our previous paper (Testamanti \& Rezaee, 2017) presented a methodology for the experimental determination of threshold $T_{2}$ cut-offs in shales, based on the analysis of cumulative NMR porosity curves which were acquired on brine-saturated, centrifuged and oven-dried core plugs (Fig. 6). In that study, two experimental threshold $\mathrm{T}_{2}$ cut-off were identified after the samples were centrifuged and oven-dried: an upper $\mathrm{T}_{2 \mathrm{U}}$ (in the range 1-2 $\mathrm{ms}$ ) and a lower $\mathrm{T}_{2 \mathrm{~L}}$ (between $0.2-0.3 \mathrm{~ms}$ ), respectively. In this current section, we will show that these threshold $T_{2}$ cut-offs could also be derived analytically, from the comparison of $T_{2}$ distributions obtained by two different NMR inversion methods. The four inversion methods applied on the synthetic $T_{2}$ data were thus used to reprocess experimental $T_{2}$ decays, which were obtained from brine-saturated shale core plugs as part of our previous

tion between the smallest and largest length scales, favors the broadening of features in 


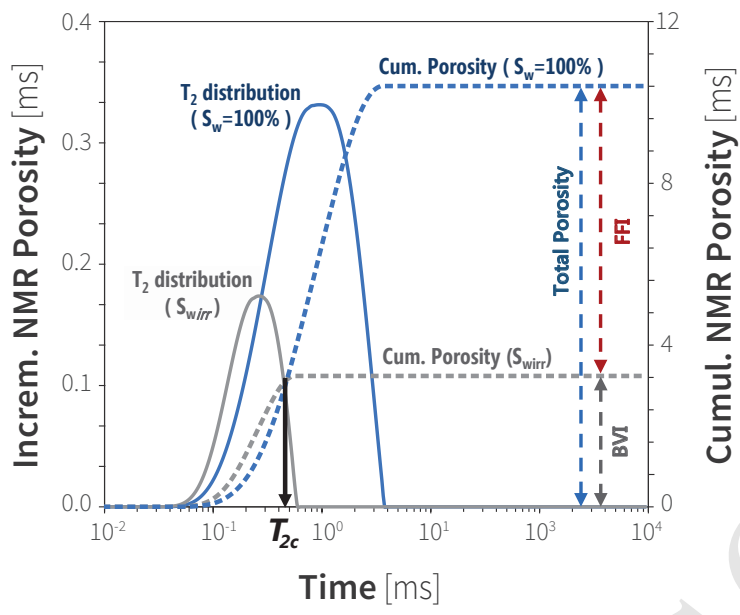

Figure 6: Methodology used in our previous study for the experimental determination of threshold $\mathrm{T}_{2}$ cut-offs $\left(\mathrm{T}_{2 \mathrm{c}}\right)$, from laboratory NMR experiments on shale samples brine-saturated $\left(\mathrm{S}_{\mathrm{w}}=100 \%\right)$ and at irreducible saturation level $\left(\mathrm{S}_{\mathrm{w}, i r r}\right.$ measured after centrifugation and oven-drying procedures). Figure modified from Testamanti \& Rezaee (2017).

and porosity values originally reported in Testamanti \& Rezaee (2017) were obtained. In addition, the raw NMR data from the brine-saturated shale samples were inverted with the BRD, UPEN and NNLS-LH/GCV algorithms. The BRD inversions were also performed in Prospa, using the Asym option in the Lexus module, which approximates the regularization weights based on the asymptotic analysis of the noise (P. Aptaker, personal communication, October 2017). Moreover, the UPEN and NNLS-LH/GCV algorithms were implemented in MATLAB (MathWorks). Prior to inversion, the raw $T_{2}$ data sizes were reduced by the SVD method.

Fig. 7 shows the inverted $T_{2}$ data from the brine-saturated shale core plugs, obtained by the BRD, UPEN and NNLS-LH/GCV methods, and compared with the $T_{2}$ distributions included in the original study (labeled $N N L S-L H / L$-curve). It can be seen from the graph that these last three methods produce similarly shaped $T_{2}$ curves, where one broad peak is displayed below $3 \mathrm{~ms}$. In contrast, an additional peak seems to emerge in some of the distributions obtained by the BRD inversion at short $T_{2}$ relaxation times.

The results obtained by BRD and NNLS-LH/L-curve inversions, from brine-saturated shale core plugs, are reproduced again in Fig. 8 for clarification purposes, along with the ${ }_{545} T_{2}$ distributions from oven-dried samples (seen in dotted gray lines). While one broad peak can be observed at early times in the original $T_{2}$ spectra (NNLS-LH) from experiments 

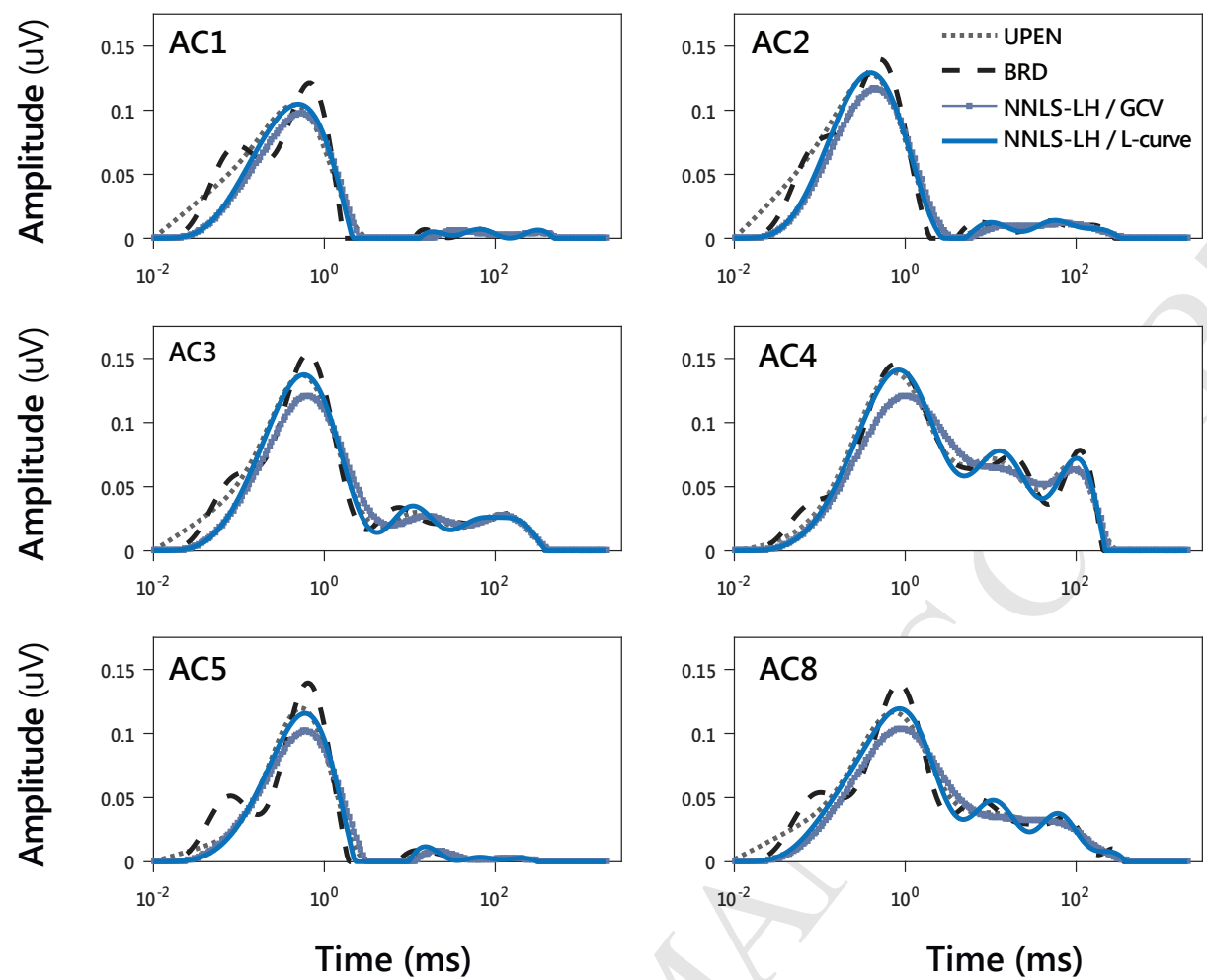

Figure 7: $\mathrm{T}_{2}$ spectra for experimental decays from saturated shale core samples, obtained by the NNLSLH/GCV, NNLS-LH/L-curve, BRD and UPEN methods.

in saturated shale core plugs, multiple peaks appear on the BRD inversion results. When comparing these two curves to the spectra from oven-dried samples, the main peak in the $T_{2}$ distribution corresponding to the NNLS-LH method can be reinterpreted as the overlapped response of at least two individual features, which emerge on the $T_{2}$ curve obtained by the BRD inversion. A portion of the fastest peak in the BRD inverted curves fall below the detection limit of the spectrometer used, however, they may still represent the real NMR signals which are partially detected during $T_{2}$ measurements. The signal close to and below $0.1 \mathrm{~ms}$ has been associated with the transverse relaxation of structural hydroxyls in clays (Fleury et al., 2013; Washburn \& Birdwell, 2013) and protons in solid organic matter (Washburn, 2014). Despite the uncertainty in the real amplitude and exact location of the additional peak distinguished by the BRD inversion around $0.1 \mathrm{~ms}$, the feature is indeed consistent with the NMR response expected in shales. Furthermore, two 
threshold $\mathrm{T}_{2}$ cut-off could be identified from the contrast of NNLH-LH and BRD inverted $T_{2}$ distributions (Fig. 8), which are also consistent with the thresholds $\mathrm{T}_{2 \mathrm{~L}}, \mathrm{~T}_{2 \mathrm{U}}$ determined experimentally in our previous study (Testamanti \& Rezaee, 2017).
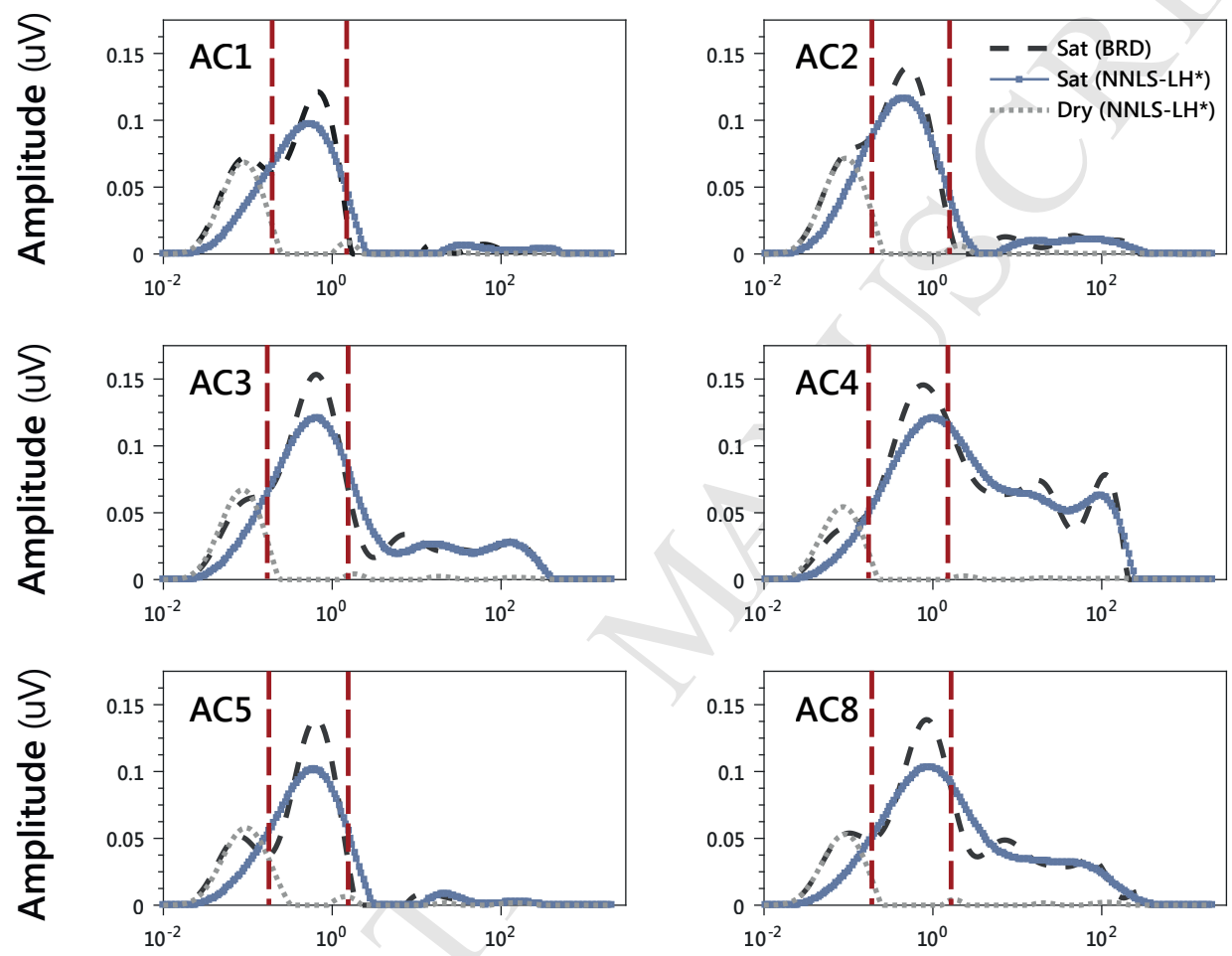

Time (ms)

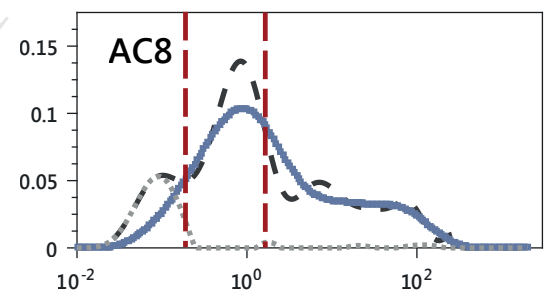

Time (ms)

Figure 8: $\mathrm{T}_{2}$ spectra from experimental decays on brine-saturated and oven-dried core samples, obtained by the NNLS-LH and BRD methods. The red dashed lines indicate the approximate locations of the threshold $\mathrm{T}_{2}$ cut-offs determined analytically, from the contrast of brine-saturated $\mathrm{T}_{2}$ distributions obtained by the different inversion methods. 


\section{Conclusion}

In this paper, various aspects of the nuclear magnetic resonance NMR technique have been examined, with a focus on one-dimensional transverse relaxation $\left(T_{2}\right)$ experiments for shale applications. The main findings of this research can be summarized as:

- Good quality laboratory NMR data can be difficult to obtain in shales. Increasing the number of scans could lead to improvements in the experimental SNR, but may also extend the duration of $T_{2}$ tests considerably. Alternatively, the SNR may be enhanced with the acquisition of a large number of data points, many of which will contain only noise. A good balance between noise and sensitivity to short $T_{2}$ components is therefore essential.

- The fast relaxation rates in shales pose unique challenges for the inversion of NMR data. Direct inversion approaches (e.g. NNLS-LH/L-curve) may produce very smooth $T_{2}$ distributions and lead to the potential loss of valuable qualitative information. Methods targeting noise level (e.g. BRD) may improve the quality of $T_{2}$ data inversion in shales, but may also bias the results.

- The shape of the $T_{2}$ distributions at short times were found to be greatly influenced by the choice of inversion algorithm, highlighting the importance of choosing a method that can yield results which are representative of the real $T_{2}$ relaxation mechanisms in shales.

- The threshold $\mathrm{T}_{2}$ cut-offs determined experimentally in our previous work are consistent with values herein identified, based on the comparison of $T_{2}$ spectra obtained by forward and indirect inversion approaches. Overall, the present findings suggest that this analytical methodology could be feasibly used to estimate $\mathrm{T}_{2}$ cut-off parameters in shales. Research into the petrophysical applications of these threshold $\mathrm{T}_{2}$ cut-off is currently underway. 


\section{Acknowledgements}

This study was supported by an Australian Government Research Training Program (RTP) Scholarship and a Curtin Research Scholarship (CRS). The authors wish to thank

Mr. Andrew Lockwood (Principal Geophysicist at Woodside Energy) for his insightful comments on an early version of this manuscript. We would also like to gratefully acknowledge the JPSE Editor-in-Chief Dr. Vural Sander Suicmez and anonymous reviewers for their constructive suggestions.

\section{Acronyms}

BRD Butler-Reeds-Dawson Algorithm.

CBW Clay Bound Water.

CPMG Carr-Purcell-Meiboom-Gill.

FID Free Induction Decay.

GCV Generalised Cross-Validation Algorithm.

LH Lawson-Hanson Algorithm.

NMR Nuclear Magnetic Resonance.

NNLS Nonnegative Least Squares Method.

PSNR Peak Signal-to-Noise Ratio.

RF Radio Frequency.

RMSE Root Mean Squared Error.

SAE Sum of Absolute Error.

SNR Signal-to-Noise Ratio.

SSI Structural Similarity Index.

SVD Singular Value Decomposition.

UPEN Uniform Penalty Algorithm.

\section{Symbols}

$B_{0}$ Static Magnetic Field.

$D$ Molecular Self-diffusion Coefficient.

$\gamma$ Gyromagnetic Ratio of Precessing Nuclei. 
615

$\lambda$ Regularization Parameter.

$M(t)$ Proton Magnetization as a Function of Time.

$M_{0}$ Proton Magnetization at Time Zero.

$N_{E}$ Number of Echoes.

$620 \rho$ Surface Relaxivity.

$S / V$ Pore Surface Area to Volume.

$T_{1}$ Longitudinal Relaxation.

$T_{2}$ Transverse Relaxation.

$T_{2} \mathbf{b}$ Transverse Relaxation of bulk fluid.

${ }_{625} T_{\text {acq }}$ Total Acquisition Time.

$T_{E}$ Echo Spacing.

$T_{W}$ Wait Time. 


\section{References}

Anovitz, L. M., \& Cole, D. R. (2015). Characterization and Analysis of Porosity and Pore Structures. Re-

views in Mineralogy and Geochemistry, 80,61-164. URL: http://rimg.geoscienceworld.org/content/ 80/1/61.short doi $10.2138 /$ rmg.2015.80.04.

Bloembergen, N., Purcell, E. M., \& Pound, R. V. (1948). Relaxation effects in nuclear magnetic resonance absorption. Physical Review, 73, 679-712. URL: https://link.aps.org/doi/10.1103/PhysRev.73.679. doi $10.1103 /$ PhysRev.73.679.

Borgia, G. C., Brown, R. J., \& Fantazzini, P. (1998). Uniform-Penalty Inversion of Multiexponential Decay Data. Journal of Magnetic Resonance, 132, 65-77. doi 10.1006/jmre.1998.1387.

Borgia, G. C., Brown, R. J. S., \& Fantazzini, P. (2000). Uniform-Penalty Inversion of Multiexponential Decay Data. Journal of Magnetic Resonance, 147, 273-285. doi 10.1006/jmre.2000.2197

Borgia, G. C., Fantazzini, P., \& Mesini, E. (1990). Water 1H spin-lattice relaxation as a fingerprint of porous media. Magnetic Resonance Imaging, 8, 435-447. doi 10.1016/0730-725X(90)90052-4

Butler, J. P., Reeds, J. A., \& Dawson, S. V. (1981). Estimating Solutions of First Kind Integral Equations with Nonnegative Constraints and Optimal Smoothing. SIAM Journal on Numerical Analysis, 18, 381-397. doi:10.1137/0718025.

Chen, S., Arro, R., Minetto, C., Georgi, D., \& Liu, C. (1998). Methods For Computing Swi And BVI From NMR Logs. In SPWLA 39th Annual Logging Symposium. Keystone, Colorado: Society of Petrophysicists and Well-Log Analysts.

Coates, G. R., Galford, J., Mardon, D., \& Marschall, D. (1998). A New Characterization Of Bulk-volume Irreducible Using Magnetic Resonance. The Log Analyst, 39.

Coates, G. R., Xiao, L., \& Prammer, M. G. (1999). NMR logging: principles and applications. Houston:

Halliburton Energy Services. URL: http://www.halliburton.com/public/lp/contents/Books_and_ Catalogs/web/NMR-Logging-Principles-and-Applications.pdf

Does, M. (2014). Multi-Exponential Relaxation Analysis (MERA) Toolbox. URL: http://www. vuiis. vanderbilt.edu/\{ $\}$ doesmd/MERA/MERA\{_\}Toolbox.html.

Dunn, K. J., Bergman, D. J., \& Latorraca, G. A. (2002). NMR in Porous Media.

Dunn, K.-J., LaTorraca, G. A., Warner, J. L., \& Bergman, D. J. (1994). On the Calculation and Interpretation of NMR Relaxation Time Distributions. doi $10.2118 / 28367-M S$

Eveleigh, L. J. (1996). Fourier transform and signal manipulation. In D. N. Rutledge (Ed.), Signal Treatment and Signal Analysis in NMR chapter 1. (pp. 1-24). Elsevier volume Vol. 18, Data Handling in Science and Technology. doi $10.1016 /$ S0922-3487(96)80038-4 
Freedman, R. (2002). Processing NMR data in the presence of coherent ringing. URL: https://patents . google.com/patent/US6838875B2/en

Gerothanassis, I. P. (1987). Methods of avoiding the effects of acoustic ringing in pulsed fourier transform nuclear magnetic resonance spectroscopy. Progress in Nuclear Magnetic Resonance Spectroscopy, 19, 267-329. doi $10.1016 / 0079-6565(87) 80005-5$.

Golub, G. H., Heath, M., \& Wahba, G. (1979). Generalized Cross-Validation as a Method for Choosing a Good Ridge Parameter. Technometrics, 21, 215-223. doi:10.2307/1268518.

Grebenkov, D. S. (2007). NMR survey of reflected Brownian motion. Reviews of Modern Physics, 79, 1077-1137. doi:10.1103/RevModPhys.79.1077

Hansen, P. C., Jensen, T. K., \& Rodriguez, G. (2007). An adaptive pruning algorithm for the discrete L-curve criterion. Journal of Computational and Applied Mathematics, 198, 483-492. doi 10.1016/j. cam.2005.09.026

Hansen, P. C., \& O'Leary, D. (1993). The Use of the L-Curve in the Regularization of Discrete Ill-Posed 685

Hürlimann, M. D. (1998). Effective Gradients in Porous Media Due to Susceptibility Differences. Journal of Magnetic Resonance, 131, 232-240. doi $10.1006 /$ jmre.1998.1364

Hürlimann, M. D., \& Venkataramanan, L. (2002). Quantitative Measurement of Two-Dimensional Distribution Functions of Diffusion and Relaxation in Grossly Inhomogeneous Fields. Journal of Magnetic 
Kenyon, W. E. (1997). Petrophysical Principles of Applications of NMR Logging. The Log Analyst, 38, $21-43$.

Kleinberg, R. L. (1999). 9. Nuclear Magnetic Resonance. In P.-z. B. T. E. M. i. t. P. S. Wong (Ed.), Methods

․ in the Physics of Porous Media (pp. 337-385). Academic Press volume 35. doi 10.1016/S0076-695X (08) $695 \quad 60420-2$

Kleinberg, R. L., Straley, C., Kenyon, W. E., Akkurt, R., \& Farooqui, S. A. (1993). Nuclear Magnetic Resonance of Rocks: T1 vs. T2. In SPE Annual Technical Conference and Exhibition. Houston, Texas, USA: Society of Petroleum Engineers. doi $10.2118 / 26470-M S$.

Lawson, C. L., \& Hanson, R. J. (1974). 26. Examples of Some Methods of Analyzing a Least Squares 700 口 Problem. In Solving Least Squares Problems chapter 26. (pp. 199-206). doi:10.1137/1.9781611971217. ch26.

McPhee, C., Reed, J., \& Zubizarreta, I. (2015). Chapter 11 - Nuclear Magnetic Resonance (NMR). In J. R. Colin McPhee, \& Z. Izaskun (Eds.), Developments in Petroleum Science (pp. 655-669). Elsevier volume Volume 64. doi 10.1016/B978-0-444-63533-4.00011-1

Miao, J., Huo, D., \& Wilson, D. L. (2008). Quantitative image quality evaluation of MR images using perceptual difference models. Medical Physics, 35, 2541-2553. doi 10.1118/1.2903207.

Mitchell, J., Chandrasekera, T. C., \& Gladden, L. F. (2012). Numerical estimation of relaxation and diffusion distributions in two dimensions. Progress in Nuclear Magnetic Resonance Spectroscopy, 62, 34-50. doi:10.1016/j.pnmrs.2011.07.002

Moody, J. B., \& Xia, Y. (2004). Analysis of multi-exponential relaxation data with very short components

using linear regularization. Journal of Magnetic Resonance, 167, 36-41. doi 10.1016/j.jmr.2003.11. 004

Morozov, V. A. (1984). Methods for Solving Incorrectly Posed Problems. New York: Springer.

Mory, A., \& Iasky, R. (1996). Stratigraphy and structure of the onshore northern Perth Basin, Western Australia. Technical Report Western Australia: Western Australia Geological Survey.

Pearce, C. E. M., Abbott, D., McDonnell, M. D., \& Stocks, N. G. (2008). Stochastic resonance: its definition, history, and debates. In Stochastic Resonance: From Suprathreshold Stochastic Resonance to 口 Stochastic Signal Quantization chapter 2. (pp. 6-46). Cambridge: Cambridge University Press. doi 10. 1017/CB09780511535239.004

Polyanin, A. D., \& Manzhirov, A. V. (2008). Handbook of Integral Equations: Second Edition. Handbooks of Mathematical Equations (2nd ed.). CRC Press. 
Prammer, M. G. (1994). NMR Pore Size Distributions and Permeability at the Well Site. doi 10.2118/ 28368-MS

Prammer, M. G., Drack, E. D., Bouton, J. C., \& Gardner, J. S. (1996). Measurements of Clay-Bound Water and Total Porosity by Magnetic Resonance Logging. Log Analyst, 37, 61-69. doi 10.2118/36522-MS

Rutledge, D. N. (1996). Signal Treatment and Signal Analysis in NMR volume 18. (1st ed.). Elsevier Science.

Saidian, M. (2015). Effect of rock composition and texture on pore size distributions in shales: Applications in low field nuclear magnetic resonance. Ph.D. thesis Colorado School of Mines.

Salimifard, B., Dick, M., Green, D., \& Ruth, D. W. (2017). Optimizing NMR Data Acquisition and Data Processing Parameters for Tight-Gas Montney Formation of Western Canada. In International Symposium of the Society of Core Analysts. Vienna, Austria: Society of Core Analysts.

Song, Y. Q. (2000). Determining Pore Sizes Using an Internal Magnetic Field. Journal of Magnetic Resonance, 143, 397-401. doi 10.1006/jmre.1999.2012.

Song, Y. Q. (2003). Using internal magnetic fields to obtain pore size distributions of porous media. Concepts in Magnetic Resonance Part A, 18A, 97-110. doi 10.1002/cmr.a.10072.

Song, Y. Q., Venkataramanan, L., \& Burcaw, L. (2005). Determining the resolution of Laplace inversion spectrum. The Journal of Chemical Physics, 122, 104104. doi 10.1063/1.1858436

Song, Y. Q., Venkataramanan, L., Hürlimann, M. D., Flaum, M., Frulla, P., \& Straley, C. (2002). T1T2 Resonance, 154, 261-268. doi 10.1006/jmre.2001.2474.

Sun, B., \& Dunn, K.-J. (2004). Methods and limitations of NMR data inversion for fluid typing. Journal

․ of Magnetic Resonance, 169, 118-128. URL: http://www.sciencedirect.com/science/article/pii/ S1090780704001028 doi $10.1016 / \mathrm{j} \cdot \mathrm{jmr} .2004 .04 .009$

745 Testamanti, M. N., \& Rezaee, R. (2017). Determination of NMR T 2 cut-off for clay bound water in shales: A case study of Carynginia Formation, Perth Basin, Western Australia. Journal of Petroleum Science and Engineering, 149, 497-503. doi:10.1016/j.petrol.2016.10.066

Tikhonov, A. N. (1963). Solution of Incorrectly Formulated Problems and the Regularization Method. Soviet Math. Dokl., 5, 1035-1038. URL: http://ci.nii.ac.jp/naid/10004315593/en/

Twomey, S. (1963). On the Numerical Solution of Fredholm Integral Equations of the First Kind by the ․ Inversion of the Linear System Produced by Quadrature. Journal of the ACM, 10, 97-101. doi 10.1145/ 321150.321157 . 
Venkataramanan, L., Gruber, F. K., Habashy, T. M., Akkurt, R., Vissapragada, B., Lewis, R. E., \&

1 Rylander, E. (2013). Methods of investigating formation samples using NMR data. URL: https: 755 //patents.google.com/patent/US9939506B2/en.

Venkataramanan, L., Song, Y. Q., \& Hürlimann, M. D. (2002). Solving Fredholm integrals of the first kind with tensor product structure in 2 and 2.5 dimensions. IEEE Transactions on Signal Processing, 50, 1017-1026. doi:10.1109/78.995059.

Wang, Z., Bovik, A. C., Sheikh, H. R., \& Simoncelli, E. P. (2004). Image quality assessment: from error 760

— visibility to structural similarity. IEEE Transactions on Image Processing, 13, 600-612. doi 10.1109/ TIP. 2003.819861.

Washburn, K. (2014). Relaxation mechanisms and shales. Concepts in Magnetic Resonance Part A, 43A, 57-78. doi $10.1002 / \mathrm{cmr} . \mathrm{a} .21302$

Washburn, K., Anderssen, E., Vogt, S., Seymour, J. D., Birdwell, J. E., Kirkland, C. M., \& Codd, S. L. 765 (2015). Simultaneous Gaussian and exponential inversion for improved analysis of shales by NMR relaxometry. Journal of Magnetic Resonance, 250, 7-16. doi:10.1016/j.jmr.2014.10.015

Washburn, K., \& Birdwell, J. (2013). Updated methodology for nuclear magnetic resonance characterization of shales. Journal of Magnetic Resonance, 233, 17-28. doi 10.1016/j·jmr.2013.04.014.

Watson, A. T., \& Chang, C. T. P. (1997). Characterizing porous media with NMR methods. Progress in 770 Nuclear Magnetic Resonance Spectroscopy, 31, 343-386. doi 10.1016/S0079-6565(97)00053-8.

Whittall, K. P. (1994). Analysis of Large One-Dimensional and Two-Dimensional Relaxation Data Sets. Journal of Magnetic Resonance, Series A, 110, 214-218. doi 10.1006/jmra.1994.1207.

Whittall, K. P. (1996). Analysis of NMR Relaxation Data. In D. N. Rutledge (Ed.), Signal Treatment and Signal Analysis in NMR chapter 3. (pp. 44-67). Elsevier volume Vol. 18, Data Handling in Science and Technology. doi 10.1016/S0922-3487(96)80040-2 


\section{Highlights}

- Evaluation of critical parameters in $\mathrm{NMR}_{2}$ data acquisition and processing for shale applications

- Sensitivity analysis to various signal acquisition parameters from $T 2$ experiments in shale cores

- Short echo spacings (TE) can improve signal quality but also enhance early experimental noise

- Inversion methods using fixed smoothing cannot resolve adjacent $T 2$ components correctly

- Inversion methods based on noise level produce better fits but may bias solutions

- The $B R D$ algorithm can differentiate the contribution from adjacent peaks at short $T_{2}$ times

- Threshold $\mathrm{T}_{2}$ cut-offs are determined analytically by using two different NMR inversion methods 